\title{
Weakly Supervised Group-Wise Model Learning Based on Discrete Optimization ${ }^{\star}$
}

\author{
René Donner ${ }^{1,2}$, Horst Wildenauer ${ }^{3}$, Horst Bischof ${ }^{2}$, and Georg Langs ${ }^{1}$ \\ 1 Computational Image Analysis and Radiology Lab, Department of Radiology, \\ Medical University of Vienna, Austria \\ rene.donner@meduniwien.ac .at \\ 2 Institute for Computer Graphics and Vision, \\ Graz University of Technology, Austria \\ 3 Pattern Recognition and Image Processing Group, \\ Vienna University of Technology, Austria
}

\begin{abstract}
In this paper we propose a method for the weakly supervised learning of sparse appearance models from medical image data based on Markov random fields (MRF). The models are learnt from a single annotated example and additional training samples without annotations. The approach formulates the model learning as solving a set of MRFs. Both the model training and the resulting model are able to cope with complex and repetitive structures. The weakly supervised model learning yields sparse MRF appearance models that perform equally well as those trained with manual annotations, thereby eliminating the need for tedious manual training supervision. Evaluation results are reported for hand radiographs and cardiac MRI slices.
\end{abstract}

\section{Introduction}

The reliable, fast segmentation of anatomical structures is a central issue in medical image analysis. It has been tackled by a number of powerful approaches. Among them are Active Shape Models / Active Appearance Models [1], Active Feature Models [2, Graph-Cuts 3, Active Contours [4, or Level-Set approaches [5]. There are two main open issues with the current approaches in model based localization and segmentation: 1. The usually limited capture range of model search, that requires some sort of initialization, making application specific heuristics necessary, and $\mathbf{2}$. the learning of the model, which is only possible with a substantial amount of user supervision. This is of particular importance for medical data, which exhibits ambiguous appearance and complex structure. In this paper we propose an approach that tackles both of these points by formulating the learning of a model in a discrete optimization framework.

The approach proposed in this paper is related to two lines of previous work 1) Group-wise registration approaches: in [6] the authors establish a mapping on a

\footnotetext{
* This work has been supported by the Austrian National Bank Fond project Computer Based Quantification of Osteoporosis and Bone Alignment, MU Vienna, TU Graz.
} 


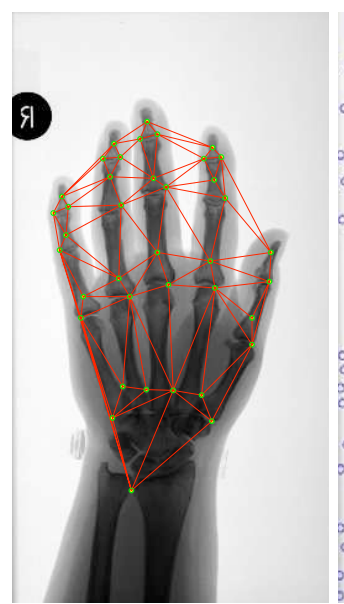

(a)

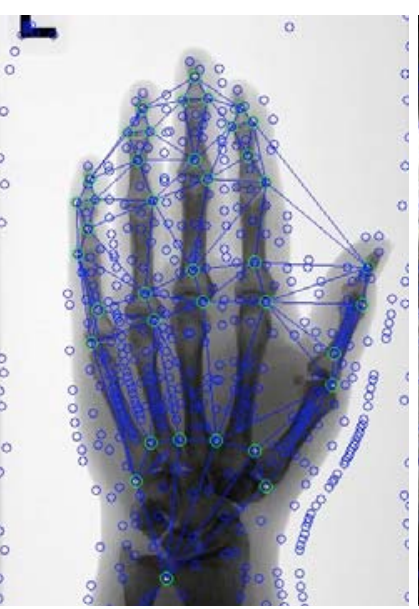

(b)

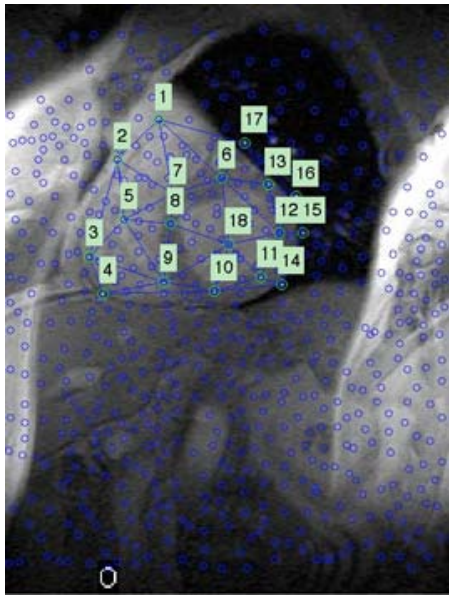

(c)

Fig. 1. (a) Example of an annotation consisting of $M$ manually selected interest points used for model building. (b) Examples of the SAM hand model match on the hand data sets. (c) Match of a SAM model trained on heart MRs on a test image. Each model node has been assigned to an interest point in the target image such that the overall MRF confidence for the match is maximized.

spherical reference manifold, [7] which employes piece-wise affine deformations to map the entire data, 8] which uses congealing to obtain a model of appearance variation from a set of images, or 9] where correspondences between sets of interest points in a population of examples are obtained. 2) Work that integrates discrete optimization for the analysis of image populations: in [10] MRFs are used as an efficient way of encoding deformations for the registration of pairs of images, in [1] Sparse MRF Appearance Models (SAMs) localize objects and structures in images with shape and appearance models based on MRFs. They use a sparse representation of the object category consisting of a statistical model of local appearance descriptors and localize geometry. The information encoded in the model and the results of the search image analysis are used to construct an MRF whose solution represents the optimal mapping of the model to the image, i. e. the best localization.

The contribution of this paper is a method that learns a sparse model of shape and appearance based on Markov random fields in a weakly supervised fashion. Instead of relying on the tedious, and potentially in-accurate annotation of the entire training data, only a single instance is annotated, to define the structure of interest. Based on this initial annotation the algorithm extends the model to the remaining training examples by iteratively including high confidence matches in the emerging model which is encoded as an MRF. The method results in a model quality equivalent to manually constructed sparse MRF appearance models, as is shown in Sec.4. We report results for two medical data sets employing combined GVFpoints [11] and Harris corners as well interest points based on superpixels [12]. 
The paper is structured as follows: In Sec.2 we outline the weakly supervised learning approach building on Sparse MRF Appearance Models. Sec.3 discusses how to employ interest point detectors based on superpixels in the SAM framework. In Sec. 4 we present the experimental evaluation of our approach, followed by conclusion and outlook in Sec.5.

\section{Model Learning by Discrete Optimization}

The learning method is based on modeling the shape and appearance variation of an object population and encoding the model's relation to an image in an MRF. The MRF's solution represents the optimal mapping of a model to an image, i. e. the best localization. During the learning phase such MRFs are solved repeatedly, optimizing the set of landmark correspondences across the images, while at the same time converging to a final model which optimally captures the properties of the entire training set. The appearance of the training examples is captured by local descriptors. The model consists of a set of landmarks, each associated with a model point descriptor $\overline{\mathbf{D}}_{m}$, and a set of edges connecting these landmarks. The edges hold the geometry information in the form of Gaussian distributions of model edge lengths $\left(\bar{l}_{a}, l_{a}^{\sigma}\right)$ and model angles $\left(\bar{\beta}_{a 1}, \bar{\beta}_{a 2}, \beta_{a 1}^{\sigma}, \beta_{a 2}^{\sigma}\right)$ as well as appearance descriptors $\overline{\mathbf{E}}_{a}$.

Shape and Appearance Model Matching by MRFs. The costs of matching of a model to an example image are encoded in the confidence function

$$
C(\mathbf{S})=\sum_{m=1 \ldots M} \mathbf{C}(m, \mathbf{S}(m))+\sum_{a=1 \ldots A} \mathbf{E}(a, \mathbf{S}(a))
$$

which consists of unary terms $\mathbf{C}$ describing the $M$ model landmarks similarities, binary terms $\mathbf{E}$ capturing the similarities of the $A$ model edges to the target edges. To estimate $\mathbf{C}$ and $\mathbf{V}$ a set of interest points $\left\{\mathbf{p}_{1}, \ldots, \mathbf{p}_{N}\right\}$ is extracted from the image with the corresponding local appearance descriptor. The MRF's solution, the so called labeling $\mathbf{S}$ maximising $C$, assigns each model node $m$ to an interest point $t$ in the target image.

The quality of a (model point $m$, target point $t$ )-match $c_{m, t}$ equals the negative distance between the local target descriptor $\mathbf{D}_{t}$ and the model point descriptor $\overline{\mathbf{D}}_{m}: c_{m, t}=-\left\|\mathbf{D}_{t}-\overline{\mathbf{D}}_{m}\right\|$. All mutual distances between model and potential target correspondences are computed, resulting in $\mathbf{C} \in \mathbb{R}^{M \times N}$ encoding the label qualities for each of the nodes. The qualities of the $A N^{2}$ edges in the model constitute $\mathbf{E} \in \mathbb{R}^{A \times N^{2}}$. The quality of an edge $e$ between two labels $n_{i}, n_{j}$ in $\mathbf{E}(a, e)=e_{\text {conf }}^{a}$ is computed by comparing its length $l_{e}$ and relative angles $\beta_{e 1}$, $\beta_{e 2}$ with the corresponding (circular) Gaussian distributions of the model edge length $\left(\bar{l}_{a}, l_{a}^{\sigma}\right)$ and model angles $\left(\bar{\beta}_{a 1}, \bar{\beta}_{a 2}, \beta_{a 1}^{\sigma}, \beta_{a 2}^{\sigma}\right)$. The confidence for the edge's appearance equals the negative distance between the edge descriptor and the model edge descriptor $\overline{\mathbf{E}}_{a}$. Each of the confidences is then normalized by operator $\mathrm{n}($.$) to a maximum of 0$ and a median of -1 (Eqs. 2,5,6). The overall confidence 
of edge $e$ representing the model edge $a$ is finally set to the minimum of the confidences for length, angles and descriptor, thus removing unlikely candidates:

$$
\begin{gathered}
e_{\text {lengthConf }}^{a}=\mathrm{n}\left(e^{-\left(l_{e}-\bar{l}_{a}\right)^{2} /\left(2 * l_{a}^{\sigma 2}\right)}-1\right) \\
e_{\text {angleConf } 1}^{a}=e^{-\left(\beta_{e 1}-\bar{\beta}_{a 1}\right)^{2} /\left(2 * \beta_{a 1}^{\sigma}{ }^{2}\right)} \\
e_{\text {angleConf } 2}^{a}=e^{-\left(\beta_{e 2}-\bar{\beta}_{a 2}\right)^{2} /\left(2 * \beta_{a 2}^{\sigma}{ }^{2}\right)} \\
e_{\text {angleConf }}^{a}=\mathrm{n}\left(\min \left(e_{\text {angleConf } 1}^{a}, e_{\text {angleConf } 2}^{a}-1\right)\right. \\
e_{\text {descriptorConf }}^{a}=\mathrm{n}\left(-\left\|E_{e}-\overline{\mathbf{E}}_{a}\right\|\right) \\
e_{\text {conf }}^{a}=\min \left(e_{\text {lengthConf }}^{a}, e_{\text {angleConf }}^{a}, e_{\text {descriptorConf }}^{a}\right)
\end{gathered}
$$

\section{Weakly Supervised Learning of the Model}

Given the sparse appearance model estimate $\mathcal{E}_{J^{k}}$ defined by the landmarks from labeling $\left\langle\mathbf{S}_{i} \mid i \in J^{k}\right\rangle$, initially derived from the manually annotated image $J^{1}=$ $\left\{j^{*}\right\}$, we can assign a confidence $C_{i}\left(\mathcal{E}_{J^{k}}\right)$ for the matching of the model estimate to the training images $i \notin J^{k}$ and thus to the corresponding target interest point (labelings $\mathbf{S}_{i}$ ). For the initial variances of the model's distributions lower bounds are used.

Based on the configuration of the landmarks we can check for the validity of the resulting labeling, allowing for the exclusion of outliers, i. e. the labelings have to conform to the model topology and the selected labels have to be unique.

Given a set of valid model confidences $C_{i}\left(\mathcal{E}_{J^{k}}\right)$ and corresponding labelings $\mathbf{S}_{i}$ the labeling

$$
\mathbf{S}_{i}^{*}=\underset{\mathbf{S}_{i}}{\operatorname{argmin}} C_{i}\left(\mathcal{E}_{J^{k}}\right)
$$

is added to the model set $J^{k}$ to compute a new model estimate $\mathcal{E}_{J^{k+1}}$ with $J^{k+1}=$ $J^{k} \cup i$. This new model estimate is again used to compute labelings for the remaining training images until $J$ comprises all training images for which valid matches can be computed, resulting in the final model estimate $\mathcal{E}^{*}$. This model is then evaluated by leave-one-out cross-validation on the whole data set (Sec.4).

\section{Capturing Local Appearance}

In this work we employ local descriptors to capture the appearance of the examples during learning. For this various approaches exist, of which we investigate two: In [1] gradient vector flow was utilized to detect interest points (GVFpoints), and describe their local appearance. The second method is based on super-pixels and can be employed for data where GVFpoints are not well suited and an even distribution of interest points is crucial. 


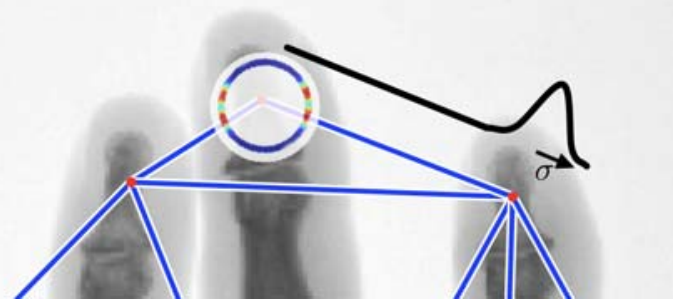

(a)

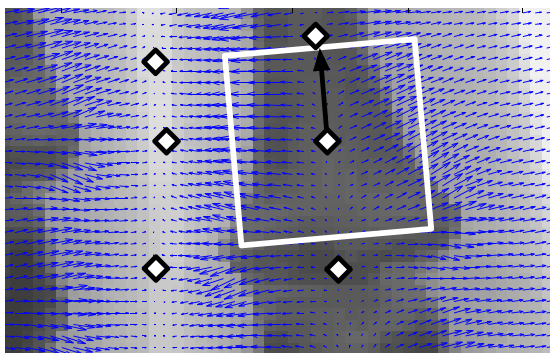

(c)

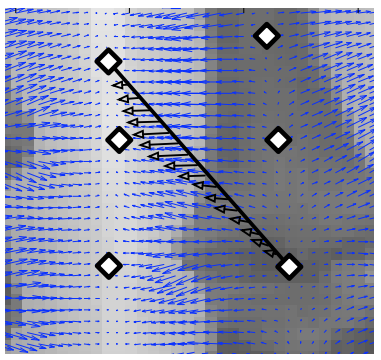

(b)

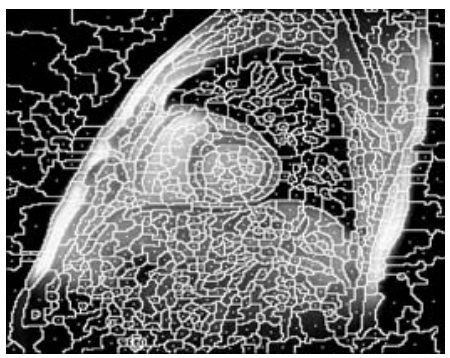

(d)

Fig. 2. (a) Illustration of the angle and length statistics learnt for a sparse appearance model node in relation to its neighbors. GVFpoints (b) point and (c) edge descriptors extracted from the GVF field. (d) Example of the watershed superpixels and the resulting interest points (centroids) for the cardiac MR data set.

1. Local Descriptors from GVF and descriptors are located at the approximate centers of homogeneous regions, thus representing rotationally oder mirror symmetric structures like the cross-sections of human organs and skeletal structures. Around each interest point patches are extracted from the vector field according to their orientation as depicted in Fig.2. To enhance the specificity of local cliques of interest points, GVFpoints can be combined with complementary information like the one derived from a Harris corner detector (Fig 1).

2. Local Descriptors from Superpixels Locally operating interest point detectors face considerable problems when confronted with medical imaging data obtained by MR or X-rays. Besides low contrast and strong noise, these images can be often characterized by a dominance of irregular, semi-local image structures. In such a setting, local intensities no longer provide stable cues for identifying discriminative features.

Recently 12 proposed Laplacian of Gaussians-based (LoG, i.e., mean curvature) watershed regions in the context of multi-scale image over-segmentation. The application of this technique leads to a complete segmentation into regularly shaped, spatially evenly distributed semi-local interest regions as shown in Fig 2(d). Note that the watersheds approximate true region boundaries very well. Also, the method is able to pick out important image structures of different sizes. Given an image $\mathbf{I}$, the LoG at position $\mathbf{x}$ is defined as $\nabla^{2} \mathbf{I}(\mathbf{x}, t)=$ 


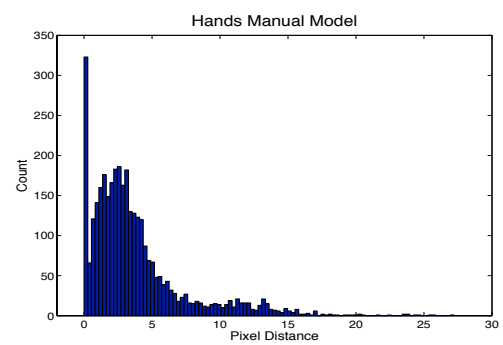

(a)

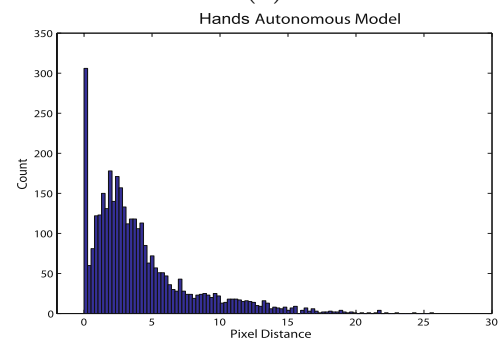

(b)

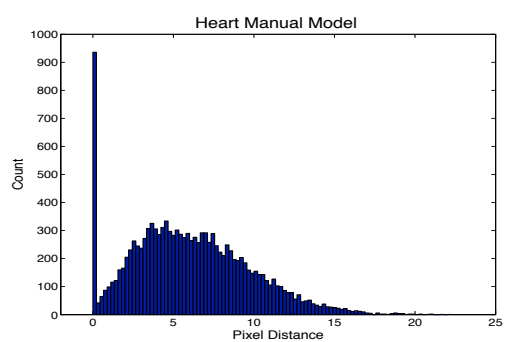

(c)

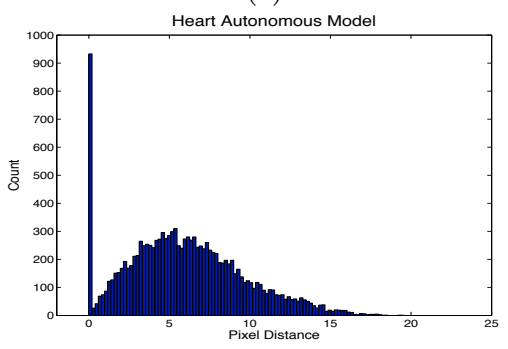

(d)

Fig. 3. Result histograms for the pixel distances of result landmarks to ground truth landmarks for the two datasets

$\mathbf{I}_{x x}(\mathbf{x}, t)+\mathbf{I}_{y y}(\mathbf{x}, t)$, where $\mathbf{I}_{x x}$ and $\mathbf{I}_{x x}$ denote the second-order partial derivatives at Gaussian blurring scale $t$. The LoG produces strong negative/positive responses for bright/dark blob and ridge-like structures. Using this, interest points are extracted as follows: 1 ) Detection of spatial extrema (seed points) in the LoG response. Discard low contrast extrema with small minimum absolute difference to adjacent pixels. 2) Segment the image into regions assigned to positive or negative mean curvature. This is achieved by applying the watershed to the negative absolute Laplacian $-\left|\nabla^{2} \mathbf{I}(\mathbf{x}, t)\right|$ using the seeds from 1. 3) Interest points are obtained as centroids of all pixels within a region. This is insensitive to small shape variations of the regions. In practice we found a dense representation of the image to give the best registration results. Due to the very moderate scale variations in our data, scale adaption was not necessary. We simply extract interest points at several predefined scales retaining the one with $\approx 800$ interest points.

\section{Experiments}

The proposed approach was evaluated on 2 medical data set:1: 1) For a set of 12 hand radiographs $(300 \times 450$ pixels $) 39$ landmarks were used consisting of both GVFpoints and interest points derived from a Harris corner detector. 2) 14 Slices

1 The implementation is available from the author's website:

http://www.cir.meduniwien.ac.at/donner-software/ 


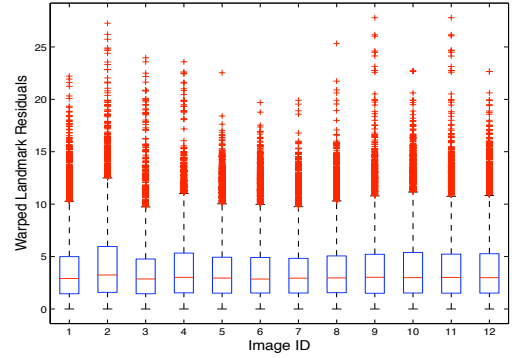

(a) Hands Data Set

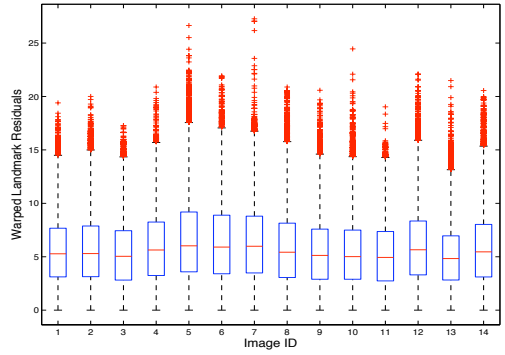

(b) Hearts Data Set

Fig. 4. Boxplot depicting the results of the leave-one-out cross-validation for different models. For each model, a different image from the training set was used as annotated start image. Note how the model's performance does not depend on the start image.

from cardiac MRs 13 with a resolution of 256x256 pixels were used together with superpixel points (Fig.1) with 18 interest points selected. This data set is very challenging to conventional interest point detectors due to low contrasts, very weak gradients and large variations throughout the set, impeding a single parameter set to yield reasonable interest points. In contrast, the super pixel based interest points proved to give reliable and descriptive locations, performing equally well on all images in the data set. The number of superpixels was $\approx 800$ for the heart data set while the total number of interest points for the hand data amounted to $\approx 1000$.

For each data set, the SAMs were run with in a leave-one-out cross validation framework a) using the manual annotations of all $T$ training images to construct the sparse appearance model and b) by employing the weakly supervised model learning approach starting from a single, manual annotation of a randomly selected image and constructing the final model as outlined in Sec.2, c) Additionally, the influence of which image was annotated was investigated.

To measure the accuracy of the method, ground truth annotations (centers of the fingers joints, inner and outer boundary of the left ventricle) were warped between all possible $n(n-1)$ image pairs according to the diffeomorphic fields imposed by the SAM matching results on the two images. The residual pixel distances between the warped landmarks to the fixed ground truth were recorded. This measure illustrates the ability of the models to capture the intrinsic structure of the data and the success of the SAMs to yield an accurate match.

Results. The results for the experiments a) and b) are displayed in Fig.3(ad). Subfigures (a) and (b) allow to compare the performance of the manual model vs. the semi-automatic approach on the hand data set, while (c,d) show the results for the cardiac MR data set. The mean/median/max values for the hand models (a) 3.77/2.80/27.22 and (b) 4.15/3.03/25.70 demonstrate the equivalent model matching capacity of both approaches. For heart MRs, (c) $6.01 / 5.71 / 22.2$ and (d) 5.98/5.70/19.54 consistently show an equal or 
better performance of the semi-automatic approach, resulting in less outliers. Fig. 4 shows that the proposed model learning approach is insensitive to the choice of the single annotated bootstrap image. This provides a high degree of robustness for practical applications. The results clearly indicate the power of the proposed weakly supervised model learning approach for SAMs to overcome the need for the manual annotation of the whole training set. The runtimes for the proposed approach are about 1 hour / $3 \mathrm{hrs}$ for the heart / hand data sets for the model learning phase, and around 10-20sec for a single localization.

\section{Conclusion and Outlook}

We present an approach for the weakly supervised learning of sparse appearance models based on MRFs. The method requires only a single annotation, and learns a model that represents appearance and geometric behavior from an training population. The method is closely related to SAMs, but yields models without the need for supervised training. It has the potential to solve the localization requirements present in many state-of-the-art image analysis approaches, being especially well suited for medical applications exhibiting complex and ambiguous structures that make manual annotation, and standard local optimization approaches, unfeasible. Together with super pixel based local descriptors this forms the prerequisite for building 3D SAMs for widespread application, as the shape annotation of $3 \mathrm{D}$ data is far more demanding and currently prohibits the wide adoption of model based approaches in this domain.

\section{References}

1. Cootes, T.F., Edwards, G.J., Taylor, C.J.: Active appearance models. IEEE Trans. PAMI 23(6), 681-685 (2001)

2. Langs, G., Peloschek, P., Donner, R., Reiter, M., Bischof, H.: Active Feature Models. In: Proc. ICPR, pp. 417-420 (2006)

3. Boykov, Y., Jolly, M.P.: Interactive graph cuts for optimal boundary \& region segmentation of objects in N-D images. In: Proc. ICCV, pp. 105-112 (2001)

4. Kass, M., Witkin, A., Terzopoulos, D.: Snakes: Active contour models. International Journal on Computer Vision 1, 321-331 (1988)

5. Paragios, N., Deriche, R.: Geodesic Active Contours and Level Sets for the Detection and Tracking of Moving Objects. IEEE PAMI 22(3) (2000)

6. Davies, R.H., Twining, C.J., Cootes, T.F., Waterton, J.C., Taylor, C.J.: 3D statistical shape models using direct optimisation of description length. In: Heyden, A., Sparr, G., Nielsen, M., Johansen, P. (eds.) ECCV 2002. LNCS, vol. 2352, pp. 3-20. Springer, Heidelberg (2002)

7. Cootes, T., Twining, C., Petrović, V., Taylor, C.: Groupwise construction of appearance models using piece-wise affine deformations. In: BMVC 2005 (2005)

8. Zöllei, L., Learned-Miller, E.G., Grimson, W.E.L., Wells, W.M.: Efficient population registration of $3 \mathrm{D}$ data. In: Liu, Y., Jiang, T.-Z., Zhang, C. (eds.) CVBIA 2005. LNCS, vol. 3765, pp. 291-301. Springer, Heidelberg (2005) 
9. Langs, G., Donner, R., Peloschek, P., Bischof, H.: Robust autonomous model learning from 2D and 3D data sets. In: Ayache, N., Ourselin, S., Maeder, A. (eds.) MICCAI 2007, Part I. LNCS, vol. 4791, pp. 968-976. Springer, Heidelberg (2007)

10. Glocker, B., Komodakis, N., Tziritas, G., Navab, N., Paragios, N.: Dense image registration through MRFs and efficient linear programming. Medical Image Analysis 12(6), 731-741 (2008)

11. Donner, R., Mičušík, B., Langs, G., Bischof, H.: Sparse MRF Appearance Models for Fast Anatomical Structure Localisation. In: Proc. BMVC (2007)

12. Wildenauer, H., Micusik, B., Vincze, M.: Efficient texture representation using multi-scale regions. In: ACCV, pp. 65-74 (2007)

13. Stegmann, M.B.: An annotated dataset of 14 cardiac MR images. Technical report, Technical University of Denmark, DTU (2002) 